\title{
Molecular Iodine: A Versatile Catalyst for the Synthesis of 4-Aryl-3-methyl-1-phenyl-1H-benzo[h]pyrazolo[3,4-b]quinoline-5,10-diones in Water
}

\author{
Liqiang Wu, ${ }^{*}$ Limin Yang, Fulin Yan, Chunguang Yang, and Lizhen Fang
}

\author{
School of Pharmacy, Xinxiang Medical University, Xinxiang, Henan 453003, P. R. China. *E-mail: wliq1974@sohu.com \\ Received November 11, 2009, Accepted February 6, 2010
}

Key Words: Benzo[h]pyrazolo[3,4- $b]$ quinoline, Molecular Iodine, 3-Methyl-1-phenyl-1H-pyrazol-5-amine, 2-Hydroxynaphthalene-1,4-dione

Multicomponent reactions (MCRs) have attracted considerable attention since they are performed without need to isolate any intermediate during their processes; may reduce time and save both energy and raw materials. ${ }^{1}$ They have merits over two-component reactions in several aspects including the simplicity of a one-pot procedure, possible structural variations and building up complex molecules.

Pyrazolo[3,4- $b]$ quinoline derivatives are used as pharmaceutical agents, ${ }^{2}$ as inhibitors of oncogenic Ras, ${ }^{3}$ and as a dopant in the multiplayer OLED fabrication. ${ }^{4}$ In the past several decades, three general strategies for the synthesis of pyrazolo [3,4-b]quinolines have been developed: 1) by the Friedlander condensation reaction of 2-aminobenzophenones and pyrazolin5-ones. ${ }^{5}$ Availability of 2-aminobenzophenones limits the range of applicability of this reaction; 2) by cyclization of 4-arylidenepyrazolin-5-ones with anilines ${ }^{6}$ or 5- $\mathrm{N}$-arylpyrazoles with aromatic aldehydes. ${ }^{7}$ The method is complicated and has a lower yield; 3 ) by a three-component one-pot reaction of aromatic aldehydes, 5-amino-3-methyl-1-phenylpyrazole and dimedone under thermal ${ }^{8}$ or microwave condition. ${ }^{9}$

Organic reactions in water have become an important research area. Many reactions have been accomplished in aqueous medium. ${ }^{10}$ Water has therefore become an attractive medium for many organic reactions, not only for the advantages concerning the avoidance of expensive, catalysts and solvents, but also for some unique reactivity and selectivity. In recent years, the use of molecular iodine in organic synthesis has received considerable attention due to powerful catalytic activity for various organic transformations. ${ }^{11}$ We now report a highly efficient procedure for the preparation of 4-aryl-3-methyl-1-phenyl$1 H$-benzo[ $h]$ pyrazolo[3,4- $b]$ quinoline-5,10-diones using $I_{2}$ as an efficient catalyst in water (Scheme 1).

Initial study was performed by the one-pot reaction of 3methyl-1-phenyl-1H-pyrazol-5-amine ( $1 \mathrm{mmol})$, benzaldehyde

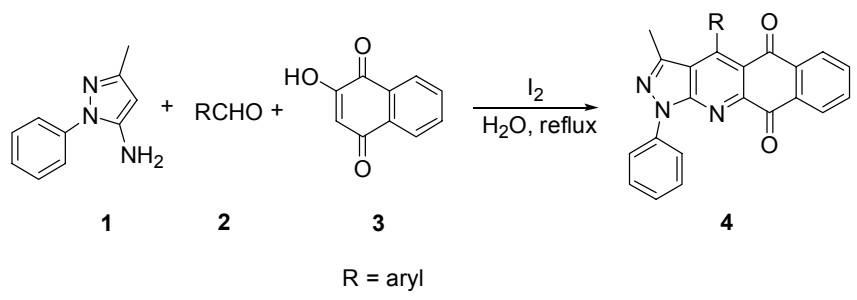

Scheme 1
(1 mmol) and 2-hydroxynaphthalene-1,4-dione (1 mmol) in

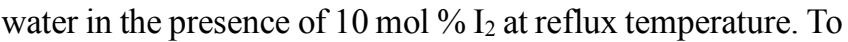
our delight, we observed the formation of 4-phenyl-3-methyl1-phenyl-1 $H$-benzo[ $h]$ pyrazolo[3,4- $b]$ quinoline-5,10-dione. Complete conversion and $92 \%$ isolated yield was obtained after 5 hours.

To optimize the amount of catalyst and the reaction temperature, the reaction of 3-methyl-1-phenyl- $1 H$-pyrazol-5-amine (1 mmol), benzaldehyde (1 mmol) and 2-hydroxynaphthalene1,4 -dione $(1 \mathrm{mmol})$ in water was selected as a model. The best result was obtained by carrying out the reaction using $10 \mathrm{~mol} \%$ $\mathrm{I}_{2}$ at reflux temperature.

Based on the optimized reaction conditions, a range of 4-aryl-3-methyl-1-phenyl-1 $H$-benzo[ $h]$ pyrazolo[3,4-b]quinoline-5,10-diones (4) was synthesized by the reaction of 3-methyl1-phenyl-1 $H$-pyrazol-5-amine $(1,1 \mathrm{mmol})$ with arylaldehydes (2, $1 \mathrm{mmol})$ and 2-hydroxynaphthalene-1,4-dione (3,1 mmol) in $\mathrm{H}_{2} \mathrm{O}$. The reaction proceeded at reflux temperature within $7 \mathrm{~h}$ in excellent yields after the addition of the cataylst $\mathrm{I}_{2}(10 \mathrm{~mol} \%)$ (Table 2). In addition, we noticed also that when this reaction was carried out with aliphatic aldehyde such as butanal or pentanal, TLC and ${ }^{1} \mathrm{H}$ NMR spectra of the reaction mixture showed a combination of starting materials and numerous products, the yield of the expected product was very poor.

Table 1. Optimization of catalyst loading and reaction temperature of one-pot synthesis of 4-phenyl-3-methyl-1-phenyl- $1 H$-benzo $[h]$ pyrazolo[3,4-b]quinoline-5,10-dione ${ }^{a}$

\begin{tabular}{ccccc}
\hline Entry & $\mathrm{I}_{2}(\mathrm{~mol} \%)$ & Time $(\mathrm{h})$ & Temp. $\left({ }^{\circ} \mathrm{C}\right)$ & ${\text { Yield }(\%)^{b}}^{b}$ \\
\hline 1 & 0 & 10 & reflux & 26 \\
2 & 5 & 8 & 80 & 62 \\
3 & 5 & 8 & 90 & 67 \\
4 & 5 & 8 & reflux & 72 \\
5 & 10 & 10 & r.t. & 56 \\
6 & 10 & 5 & 50 & 62 \\
7 & 10 & 5 & 80 & 75 \\
8 & 10 & 5 & 90 & 86 \\
9 & 10 & 5 & reflux & 92 \\
10 & 15 & 5 & 90 & 86 \\
11 & 15 & 5 & reflux & 90 \\
12 & 20 & 5 & reflux & 91
\end{tabular}

${ }^{a}$ Reaction conditions: 3-methyl-1-phenyl-1 $H$-pyrazol-5-amine (1 mmol); benzaldehyde (1 mmol); 2-hydroxynaphthalene-1,4-dione (1 mmol); $\mathrm{H}_{2} \mathrm{O}$ $(10 \mathrm{~mL}) .{ }^{5}$ Isolated yield. 
In general, iodine is reaction with water to afford strong acid. Therefore the following reaction mechanism is suggested. First, the condensation of 3-methyl-1-phenyl- $1 H$-pyrazol-5-amine 1 and 2-hydroxynaphthalene-1,4-dione 3 gave the intermediate

Table 2. Synthesis of 4-aryl-3-methyl-1-phenyl-1 $H$-benzo[ $h]$ pyrazolo[3,4- $b$ ]quinoline-5,10-diones ${ }^{a}$

\begin{tabular}{ccccc}
\hline Entry & $\mathrm{R}$ & Time $(\mathrm{h})$ & Product & Yield $(\%)^{b}$ \\
\hline 1 & $\mathrm{C}_{6} \mathrm{H}_{5}$ & 5 & $\mathbf{4 a}$ & 92 \\
2 & $4-\mathrm{Cl}-\mathrm{C}_{6} \mathrm{H}_{4}$ & 6 & $\mathbf{4 b}$ & 93 \\
3 & $4-\mathrm{MeO}-\mathrm{C}_{6} \mathrm{H}_{4}$ & 5 & $\mathbf{4 c}$ & 90 \\
4 & $4-\mathrm{Me}-\mathrm{C}_{6} \mathrm{H}_{4}$ & 5 & $\mathbf{4 d}$ & 88 \\
5 & $4-\mathrm{NO}_{2}-\mathrm{C}_{6} \mathrm{H}_{4}$ & 6 & $\mathbf{4 e}$ & 91 \\
6 & $4-\mathrm{C}_{6} \mathrm{H}_{4}$ & 6 & $\mathbf{4 f}$ & 94 \\
7 & $3-\mathrm{NO}_{2}-\mathrm{C}_{6} \mathrm{H}_{4}$ & 7 & $\mathbf{4 g}$ & 87 \\
8 & $2-\mathrm{Cl}_{6} \mathrm{C}_{6} \mathrm{H}_{4}$ & 5 & $\mathbf{4 h}$ & 85 \\
9 & $3,4-\mathrm{Cl}_{2}-\mathrm{C}_{6} \mathrm{H}_{3}$ & 7 & $\mathbf{4 i}$ & 89 \\
\hline
\end{tabular}

${ }^{a}$ Reaction conditions: 3-methyl-1-phenyl-1 $H$-pyrazol-5-amine (1 mmol); arylaldehyde (1 mmol); 2-hydroxynaphthalene-1,4-dione (1 mmol); $\mathrm{I}_{2}$ (10 mol \%); $\mathrm{H}_{2} \mathrm{O}(10 \mathrm{~mL})$; reflux. ${ }^{b}$ Isolated yield. product 5 . The addition of 5 to aldehyde 2 then furnished the intermediate product $\mathbf{6}$, which on intermolecular cyclization and dehydration gave rise to 7 . In the last step, the intermediateproduct 7 aromatized by air-oxidation to product 4 .

In order to determine air-oxidation proceeded in the reaction, multicomponent condensation reaction of 3-methyl-1-phenyl$1 H$-pyrazol-5-amine, 4-chorobenzaldehyde and 2-hydroxynaphthalene-1,4-dione in the presence of molecular iodine was selected as a model. Compound $\mathbf{7 b}$ was a principal product in nitrogen atmosphere. The compound $\mathbf{7 b}$ was easily oxidized by air bubbling, forming the fully aromatic product $\mathbf{4 b}$ (Scheme 3 ).

In summary, an efficient methodology for the synthesis of 4-aryl-3-methyl-1-phenyl- $1 H$-benzo[ $h]$ pyrazolo[3,4- $b]$ quinoline-5,10-diones has been developed. To our best knowledge, this is the first report for the synthesis of these compounds by multicomponent condensation of 3-methyl-1-phenyl-1H-pyrazol-5-amine, aldehydes and 2-hydroxynaphthalene-1,4-dione in the presence of molecular iodine as a catalyst in water. The simple experimental procedure, utilization of an inexpensive and readily available catalyst, and excellent yields are the advantages of the present method.<smiles>CCC1=C(C)C(=O)C=C(O)C1=O</smiles><smiles>C1CCC1</smiles><smiles></smiles><smiles>C[BiH]</smiles><smiles>O=C1CC(=O)c2ccccc2C1=O</smiles><smiles>[R]C1C(C)=C2C(=O)c3ccccc3C(=O)C(Nc3c(C)nn(-c4ccccc4)c3C)=C21</smiles><smiles>Cc1cc(N)n(-c2ccccc2)n1</smiles>

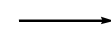<smiles>Cc1cc(N=C2CC(=O)c3ccccc3C2=O)n(-c2ccccc2)n1</smiles>

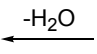

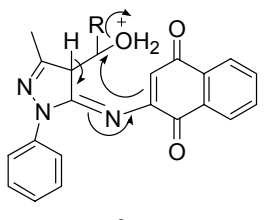<smiles>CC=O</smiles><smiles>Cc1cc(NC2=CC(=O)c3ccccc3C2=O)n(-c2ccccc2)n1</smiles>

Scheme 2

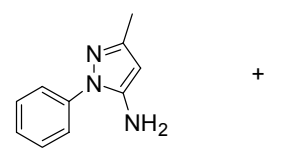<smiles>O=Cc1ccc(Cl)cc1</smiles><smiles>O=C1C=C(O)C(=O)c2ccccc21</smiles>

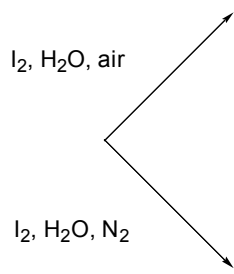<smiles>Cc1nn(-c2ccccc2)c2nc3c(c(-c4ccc(Cl)cc4)c12)C(=O)c1ccccc1C3=O</smiles><smiles>Cc1nn(-c2ccccc2)c2c1C(c1ccc(Cl)cc1)C1=C(N2)C(=O)c2ccccc2C1=O</smiles>

Scheme 3 


\section{Experimental Section}

4-Aryl-3-methyl-1-phenyl-1 $H$-benzo $[h]$ pyrazolo $[3,4-b]$ quinoline-5,10-diones (4a-4i). A mixture of 3-methyl-1-phenyl$1 \mathrm{H}$-pyrazol-5-amine ( $1 \mathrm{mmol})$, aldehyde ( $1 \mathrm{mmol}), 2$-hydroxynaphthalene-1,4-dione $(1 \mathrm{mmol})$ and $\mathrm{I}_{2}(0.1 \mathrm{mmol})$ in $\mathrm{H}_{2} \mathrm{O}(10$ $\mathrm{mL}$ ) was heated at reflux temperature for the appropriate time. The reaction was monitored by TLC. After completion, the mixture was treated with aqueous $\mathrm{Na}_{2} \mathrm{~S}_{2} \mathrm{O}_{3}$ solution, extracted with $\mathrm{CH}_{2} \mathrm{Cl}_{2}(2 \times 10 \mathrm{~mL})$. The extract was dried over sodium sulfate, filtered and solvent was evaporated in vacuo. Products 4 were purified by recrystallizing from ethanol.

4-Phenyl-3-methyl-1-phenyl-1 $\boldsymbol{H}$-benzo $[\boldsymbol{h}]$ pyrazolo $[3,4-b]$ quinoline-5,10-dione (4a): Yellow crystals, mp $266 \sim 267{ }^{\circ} \mathrm{C}$; ${ }^{1} \mathrm{H} \mathrm{NMR}\left(\mathrm{CDCl}_{3}, 400 \mathrm{MHz}\right) \delta 8.82(\mathrm{~d}, 1 \mathrm{H}, J=8.0 \mathrm{~Hz}), 8.31$ $(\mathrm{d}, 2 \mathrm{H}, J=7.6 \mathrm{~Hz}), 8.16(\mathrm{~d}, 1 \mathrm{H}, J=7.6 \mathrm{~Hz}), 7.88-7.84(\mathrm{~m}, 1 \mathrm{H})$, 7.64-7.52 (m, 6H), 7.43-7.39 (m, 1H), 7.32-7.30 (m, 2H), 1.95 $(\mathrm{s}, 3 \mathrm{H}) ;{ }^{13} \mathrm{C} \mathrm{NMR}\left(\mathrm{CDCl}_{3}, 100 \mathrm{MHz}\right) \delta 180.5,179.9,153.7$, 152.2, 150.5, 146.5, 138.6, 137.3, 136.0, 135.9, 131.7, 131.3, 129.2, 129.1, 128.4, 127.2, 127.0, 126.5, 121.3, 119.8, 117.0, 14.3; Anal. calcd for $\mathrm{C}_{27} \mathrm{H}_{17} \mathrm{~N}_{3} \mathrm{O}_{2}$ : C 78.06, H 4.12, N 10.11; found: C 78.19, H 4.06, N10.08.

4-(p-Chloro-phenyl)-3-methyl-1-phenyl-1 H-benzo[ $h]$ pyrazolo[3,4-b]quinoline-5,10-dione (4b): Yellow crystals, mp 243 $245^{\circ} \mathrm{C} ;{ }^{1} \mathrm{H} \mathrm{NMR}\left(\mathrm{CDCl}_{3}, 400 \mathrm{MHz}\right) \delta 8.80(\mathrm{~d}, 1 \mathrm{H}, J=8.0 \mathrm{~Hz})$, $8.30(\mathrm{~d}, 2 \mathrm{H}, J=8.0 \mathrm{~Hz}), 8.15(\mathrm{~d}, 1 \mathrm{H}, J=7.6 \mathrm{~Hz}), 7.87-7.83$ $(\mathrm{m}, 1 \mathrm{H}), 7.64-7.51(\mathrm{~m}, 5 \mathrm{H}), 7.43-7.40(\mathrm{~m}, 1 \mathrm{H}), 7.28-7.24(\mathrm{~m}$, 2H), $2.00(\mathrm{~s}, 3 \mathrm{H}) ;{ }^{13} \mathrm{C} \mathrm{NMR}\left(\mathrm{CDCl}_{3}, 100 \mathrm{MHz}\right) \delta 180.3,179.9$, $153.8,150.8,150.5,146.2,138.5,137.1,136.0,134.6,134.3$, 131.6, 131.4, 129.3, 129.2, 129.1, 128.7, 128.5, 127.2, 126.7, 121.4, 121.0, 119.7, 116.8, 14.5; Anal. calcd for $\mathrm{C}_{27} \mathrm{H}_{16} \mathrm{ClN}_{3} \mathrm{O}_{2}$ : C 72.08, H 3.58, N 9.34; found: C 72.35, H 3.39, N 9.50.

4-(p-Methoxyl-phenyl)-3-methyl-1-phenyl-1H-benzo[ $h]$ pyrazolo[3,4-b]quinoline-5,10-dione (4c): Yellow crystals, mp $274 \sim 275^{\circ} \mathrm{C} ;{ }^{1} \mathrm{H} \mathrm{NMR}\left(\mathrm{CDCl}_{3}, 400 \mathrm{MHz}\right) \delta 8.82(\mathrm{~d}, 1 \mathrm{H}, J=$ $8.0 \mathrm{~Hz}), 8.32(\mathrm{~d}, 2 \mathrm{H}, J=8.0 \mathrm{~Hz}), 8.16(\mathrm{~d}, 1 \mathrm{H}, J=8 \mathrm{~Hz}), 7.88-$ $7.84(\mathrm{~m}, 1 \mathrm{H}), 7.64-7.60(\mathrm{~m}, 3 \mathrm{H}), 7.43-7.39(\mathrm{~m}, 1 \mathrm{H}), 7.23(\mathrm{~d}$, $2 \mathrm{H}, J=8.4 \mathrm{~Hz}$ ), 7.06 (d, 2H, $J=8.4 \mathrm{~Hz}), 3.93$ (s, 3H), 2.03 (s, $3 \mathrm{H}) ;{ }^{13} \mathrm{C} \mathrm{NMR}\left(\mathrm{CDCl}_{3}, 100 \mathrm{MHz}\right) \delta 180.8,180.3,159.8,153.8$, 152.4, 150.5, 146.6, 138.6, 137.4, 136.0, 131.6, 131.3, 129.2, 129.0, 128.6, 127.7, 127.2, 126.5, 121.4, 120.2, 117.3, 113.9, 55.3, 14.6; Anal. calcd for $\mathrm{C}_{28} \mathrm{H}_{19} \mathrm{~N}_{3} \mathrm{O}_{3}: \mathrm{C} 75.49, \mathrm{H} 4.30, \mathrm{~N} 9.43$; found: C 75.41, H 4.33, N 9.51.

4-(p-Methyl-phenyl)-3-methyl-1-phenyl-1 $H$-benzo[ $h]$ pyrazolo[3,4-b]quinoline-5,10-dione (4d): Yellow crystals, mp $276 \sim 277^{\circ} \mathrm{C} ;{ }^{1} \mathrm{H} \mathrm{NMR}\left(\mathrm{CDCl}_{3}, 400 \mathrm{MHz}\right) \delta 8.82(\mathrm{~d}, 1 \mathrm{H}, J=$ $8.0 \mathrm{~Hz}), 8.31(\mathrm{~d}, 2 \mathrm{H}, J=8.0 \mathrm{~Hz}), 8.15(\mathrm{~d}, 1 \mathrm{H}, J=7.6 \mathrm{~Hz}), 7.85$ $(\mathrm{t}, 1 \mathrm{H}, J=7.8 \mathrm{~Hz}), 7.63-7.60(\mathrm{~m}, 3 \mathrm{H}), 7.41(\mathrm{t}, 1 \mathrm{H}, J=7.2 \mathrm{~Hz})$, $7.33(\mathrm{~d}, 2 \mathrm{H}, J=7.6 \mathrm{~Hz}), 7.18(\mathrm{~d}, 2 \mathrm{H}, J=7.6 \mathrm{~Hz}), 2.49(\mathrm{~s}, 3 \mathrm{H})$, $1.99(\mathrm{~s}, 3 \mathrm{H}) ;{ }^{13} \mathrm{C} \mathrm{NMR}\left(\mathrm{CDCl}_{3}, 100 \mathrm{MHz}\right) \delta 180.7,180.1,153.7$, 152.6, 150.5, 146.7, 138.6, 138.2, 137.4, 135.9, 132.8, 131.6, 131.3, 129.2, 129.1, 129.0, 127.2, 126.9, 126.5, 121.4, 120.0, 117.1, 21.5, 14.4; Anal. calcd for $\mathrm{C}_{28} \mathrm{H}_{19} \mathrm{~N}_{3} \mathrm{O}_{2}$ : C 78.31, H 4.46, N 9.78; found: C 78.50, H 4.42, N 9.82.

4-(p-Nitro-phenyl)-3-methyl-1-phenyl-1 $H$-benzo[h]pyrazolo[3,4-b]quinoline-5,10-dione (4e): Yellow crystals, mp $326 \sim 328{ }^{\circ} \mathrm{C} ;{ }^{1} \mathrm{H} \mathrm{NMR}\left(\mathrm{CDCl}_{3}, 400 \mathrm{MHz}\right) \delta 8.82(\mathrm{~d}, 1 \mathrm{H}, J=$ $8.0 \mathrm{~Hz}), 8.42(\mathrm{~d}, 2 \mathrm{H}, J=8.4 \mathrm{~Hz}), 8.30(\mathrm{~d}, 2 \mathrm{H}, J=8.0 \mathrm{~Hz}), 8.18$ $(\mathrm{d}, 1 \mathrm{H}, J=7.6 \mathrm{~Hz}), 7.88(\mathrm{t}, 1 \mathrm{H}, J=7.8 \mathrm{~Hz}), 7.67-7.62(\mathrm{~m}, 3 \mathrm{H})$, $7.51(\mathrm{~d}, 2 \mathrm{H}, J=8.8 \mathrm{~Hz}), 7.44(\mathrm{t}, 1 \mathrm{H}, J=7.4 \mathrm{~Hz}), 1.97(\mathrm{~s}, 3 \mathrm{H})$; ${ }^{13} \mathrm{C} \mathrm{NMR}\left(\mathrm{CDCl}_{3}, 100 \mathrm{MHz}\right) \delta 179.8,179.6,153.9,150.5$, 149.2, 147.9, 145.7, 143.1, 138.4, 136.9, 136.2, 131.7, 131.6, 129.4, 129.3, 128.2, 127.3, 126.9, 123.8, 121.5, 119.2, 116.1, 14.5; Anal. calcd for $\mathrm{C}_{27} \mathrm{H}_{16} \mathrm{~N}_{4} \mathrm{O}_{4}$ : C 70.43, H 3.50, N 12.17; found: C 70.48, H 3.38, N 12.25.

4-(p-Fluoro-phenyl)-3-methyl-1-phenyl-1H-benzo[h]pyrazolo[3,4-b]quinoline-5,10-dione (4f): Yellow crystals, mp $282 \sim 283{ }^{\circ} \mathrm{C} ;{ }^{1} \mathrm{H}$ NMR (DMSO- $\left.d_{6}, 400 \mathrm{MHz}\right) \delta 8.77(\mathrm{~d}, 1 \mathrm{H}$, $J=8.0 \mathrm{~Hz}), 8.30(\mathrm{~d}, 2 \mathrm{H}, J=7.6 \mathrm{~Hz}), 8.05(\mathrm{~d}, 1 \mathrm{H}, J=7.2 \mathrm{~Hz})$, 7.98-7.94 (m, 1H), 7.74-7.66 (m, 3H), 7.44-7.36 (m, 5H), $1.90(\mathrm{~s}$, $3 \mathrm{H}) ;{ }^{13} \mathrm{C} \mathrm{NMR}\left(\mathrm{CDCl}_{3}, 100 \mathrm{MHz}\right) \delta 180.5,180.0,164.1,161.6$, 153.8, 151.1, 150.2, 146.3, 138.6, 137.2, 136.0, 131.7, 131.6, 131.4, 129.3, 129.2, 129.1, 129.0, 128.9, 127.2, 126.7, 121.4, 119.9, 117.0, 115.7, 115.5, 14.4; Anal. calcd for $\mathrm{C}_{27} \mathrm{H}_{16} \mathrm{FN}_{3} \mathrm{O}_{2}$ : C 74.82, H 3.72, N 9.69; found: C 75.02, H 3.68, N 9.76.

4-(m-Nitro-phenyl)-3-methyl-1-phenyl-1 H-benzo[ $h]$ pyrazolo[3,4-b]quinoline-5,10-dione (4g): Yellow crystals, mp $288 \sim 289{ }^{\circ} \mathrm{C} ;{ }^{1} \mathrm{H}$ NMR $\left(\mathrm{CDCl}_{3}, 400 \mathrm{MHz}\right) \delta 8.83(\mathrm{~d}, 1 \mathrm{H}, J=$ $8.0 \mathrm{~Hz}), 8.41(\mathrm{~d}, 1 \mathrm{H}, J=8.4 \mathrm{~Hz}), 8.30(\mathrm{~d}, 2 \mathrm{H}, J=8.0 \mathrm{~Hz}), 8.24$ $(\mathrm{s}, 1 \mathrm{H}), 8.18(\mathrm{~d}, 1 \mathrm{H}, J=7.6 \mathrm{~Hz}), 7.89(\mathrm{t}, 1 \mathrm{H}, J=7.2 \mathrm{~Hz}), 7.75$ $(\mathrm{t}, 1 \mathrm{H}, J=7.8 \mathrm{~Hz}), 7.69-7.61(\mathrm{~m}, 4 \mathrm{H}), 7.44(\mathrm{t}, 1 \mathrm{H}, J=14.8 \mathrm{~Hz})$, 1.97 (s, 3H); ${ }^{13} \mathrm{C} \mathrm{NMR}\left(\mathrm{CDCl}_{3}, 100 \mathrm{MHz}\right) \delta 179.9,179.7,153.9$, 150.6, 148.7, 148.2, 145.6, 138.4, 137.7, 136.9, 136.2, 133.3, 131.7, 131.6, 129.6, 129.3, 129.2, 127.3, 126.9, 123.4, 122.4, 121.5, 119.4, 116.5, 14.6; Anal. calcd for $\mathrm{C}_{27} \mathrm{H}_{16} \mathrm{~N}_{4} \mathrm{O}_{4}$ : C 70.43, H 3.50, N 12.17; found: C 70.54, H 3.32, N 12.21.

4-(o-Chloro-phenyl)-3-methyl-1-phenyl-1 $H$-benzo[ $h]$ pyrazolo[3,4-b]quinoline-5,10-dione (4h): Yellow crystals, mp $229 \sim 230{ }^{\circ} \mathrm{C} ;{ }^{1} \mathrm{H} \mathrm{NMR}\left(\mathrm{CDCl}_{3}, 400 \mathrm{MHz}\right) \delta 8.84(\mathrm{~d}, 1 \mathrm{H}, J=$ $8.0 \mathrm{~Hz}), 8.33$ (d, $2 \mathrm{H}, J=8.0 \mathrm{~Hz}), 8.19(\mathrm{~d}, 1 \mathrm{H}, J=7.6 \mathrm{~Hz}), 7.89-$ $7.85(\mathrm{~m}, 1 \mathrm{H}), 7.65-7.40(\mathrm{~m}, 7 \mathrm{H}), 7.25-7.23(\mathrm{~m}, 1 \mathrm{H}), 2.00(\mathrm{~s}, 3 \mathrm{H})$; ${ }^{13} \mathrm{C} \mathrm{NMR}\left(\mathrm{CDCl}_{3}, 100 \mathrm{MHz}\right) \delta 179.9,179.3,153.8,150.8$, 148.5, 146.2, 138.6, 137.2, 136.0, 135.1, 131.7, 131.6, 131.4, $129.9,129.5,129.3,129.2,128.3,127.2,127.0,126.6,121.4$, 119.6, 116.5, 13.6; Anal. calcd for $\mathrm{C}_{27} \mathrm{H}_{16} \mathrm{ClN}_{3} \mathrm{O}_{2}$ : C 72.08, $\mathrm{H}$ 3.58, N 9.34; found: C 72.19, H 3.70, N 9.28.

4-(3,4-Dichloro-phenyl)-3-methyl-1-phenyl-1 $H$-benzo $[h]$ pyrazolo[3,4-b]quinoline-5,10-dione (4i): Yellow crystals, $\mathrm{mp}$ $269 \sim 270{ }^{\circ} \mathrm{C} ;{ }^{1} \mathrm{H} \mathrm{NMR}\left(\mathrm{CDCl}_{3}, 400 \mathrm{MHz}\right) \delta 8.78(\mathrm{~d}, 1 \mathrm{H}, J=$ $8.0 \mathrm{~Hz}), 8.28(\mathrm{~d}, 2 \mathrm{H}, J=8.0 \mathrm{~Hz}), 8.15(\mathrm{~d}, 1 \mathrm{H}, J=7.6 \mathrm{~Hz}), 7.87-$ $7.83(\mathrm{~m}, 1 \mathrm{H}), 7.63-7.59(\mathrm{~m}, 4 \mathrm{H}), 7.43-7.40(\mathrm{~m}, 2 \mathrm{H}), 7.16(\mathrm{~d}$, $1 \mathrm{H}, J=8.0 \mathrm{~Hz}), 2.03(\mathrm{~s}, 3 \mathrm{H}) ;{ }^{13} \mathrm{C} \mathrm{NMR}\left(\mathrm{CDCl}_{3}, 100 \mathrm{MHz}\right) \delta$ $179.9,179.5,153.7,150.4,148.9,145.9,138.4,136.9,136.1$, $135.7,132.8,131.5,130.5,129.2,129.0,127.2,126.8,126.6$, 121.3, 119.4, 116.5, 14.7; Anal. calcd for $\mathrm{C}_{27} \mathrm{H}_{15} \mathrm{Cl}_{2} \mathrm{~N}_{3} \mathrm{O}_{2}$ : C 66.96, H 3.12, N 8.68; found: C 67.03, H 3.20, N 8.58.

1,4-Dihydrogen-4-(p-chloro-phenyl)-3-methyl-1-phenyl$1 \boldsymbol{H}$-benzo[h]pyrazolo[3,4-b]quinoline-5,10-dione (7b). In nitrogen atmosphere, a mixture of 3-methyl-1-phenyl-1H-pyrazol5-amine (1 mmol), 4-chorobenzaldehyde (140 mg, $1 \mathrm{mmol})$, 2-hydroxynaphthalene-1,4-dione $(1 \mathrm{mmol})$ and $\mathrm{I}_{2}(0.1 \mathrm{mmol})$ in $\mathrm{H}_{2} \mathrm{O}(10 \mathrm{~mL})$ was stirred at room temperature for $5 \mathrm{~h}$. The reaction was monitored by TLC. After completion, the mixture was treated with aqueous $\mathrm{Na}_{2} \mathrm{~S}_{2} \mathrm{O}_{3}$ solution, extracted with $\mathrm{CH}_{2} \mathrm{Cl}_{2}(2 \times 10 \mathrm{~mL})$. The extract was dried over sodium sulfate, filtered and solvent was evaporated in vacuo. The residue was 
purified by silica gel column chromatography using $\mathrm{CHCl}_{3}$ as eluent to give $7 \mathbf{b}$ as a brownish red solid (360 mg, 80\%); mp $252 \sim 253{ }^{\circ} \mathrm{C} ;{ }^{1} \mathrm{H} \mathrm{NMR}\left(\mathrm{CDCl}_{3}, 400 \mathrm{MHz}\right) \delta 8.12(\mathrm{t}, 2 \mathrm{H}, J=$ $9.4 \mathrm{~Hz}), 7.80-7.32(\mathrm{~m}, 10 \mathrm{H}), 7.06(\mathrm{t}, 2 \mathrm{H}, J=8.4 \mathrm{~Hz}), 5.45$ (s, $1 \mathrm{H}), 2.03$ (s, 3H); Anal. calcd for $\mathrm{C}_{27} \mathrm{H}_{18} \mathrm{ClN}_{3} \mathrm{O}_{2}: \mathrm{C} 71.76, \mathrm{H}$ 4.01, N 9.30; found: C 71.84, H 3.96, N 9.35.

Acknowledgments. We are pleased to acknowledge the financial support from Xinxiang Medical University.

\section{References}

1. Devi, I.; Bhuyan, P. J. Tetrahedron Lett. 2004, 45, 8625.

2. El-Sayed, O. A.; Aboul-Enein, H. Y. Arch. Pharm. 2001, 334, 117.

3. Wolin, R.; Wang, D.; Kelly, J.; Afonso, A.; James, L.; Kirschmeier, P.; Mcphail, A. T. Bioorg. Med. Chem. Lett. 1996, 6, 195.
4. He, Z.; Milburn, G. H. W.; Baldwin, K. J.; Smith, D. A.; Danel, A.; Tomasik, P. J. Lumin. 2000, 86, 1.

5. Tomasik, P.; Tomasik, D.; Abramovitch, R. A. J. Heterocycl. Chem. 1983, 20, 1539.

6. Chaczatrian, K.; Chaczatrian, G.; Danel, A.; Tomasik, P. ARKIVOC 2001, (vi), 63.

7. Danel, A.; Chaczatrian, K.; Tomasik, P. ARKIVOC 2000, (i), 51.

8. Quiroga, J.; Insuasty, B.; Saitz, C.; Jullian, C. J. Heterocycl. Chem. 1998, 35, 575 .

9. Hua, G. P.; Xu, J. N.; Tu, S. J.; Wang, Q.; Zharlg, J. P.; Zhu, X. T.; Li, T. J.; Zhu, S. L.; Zhang, X. J. Chin. Org. Chem. 2005, 25, 1610. 10. Lindstrom, U. M. Chem. Rev. 2002, 102, 2751.

11. (a) Das, B.; Ravikanth, B.; Ramu, R.; Laxminarayana, K.; Rao, B. V. J. Mol. Catal. A: Chem. 2006, 255, 74. (b) Kidwai, M.; Bansal, V.; Mothsra, P.; Saxena, S.; Somvanshi, R. K.; Dey, S.; Singh, T. P. J. Mol. Catal. A: Chem. 2007, 268, 76. (c) Bhosale, R. S.; Magar, C. V.; Solanke, K. S.; Mane, S. B.; Choudhary, S. S.; Pawar, R. P. Synth. Commun. 2007, 37, 4353. 